\title{
ALMOST PERIODIC SYNCHRONIZATION OF FUZZY CELLULAR NEURAL NETWORKS WITH TIME-VARYING DELAYS VIA STATE-FEEDBACK AND IMPULSIVE CONTROL
}

\author{
YONGKUn LI $^{a, *}$, HUIMEI WANG ${ }^{b}$, XiAOFANG MENG ${ }^{a}$ \\ ${ }^{a}$ Department of Mathematics \\ Yunnan University, Kunming, Yunnan 650091, People's Republic of China \\ e-mail: ykliedynu.edu.cn \\ ${ }^{b}$ Department of Mathematics \\ Kunming University, Kunming, Yunnan 650214, People’s Republic of China
}

\begin{abstract}
In this paper, we are concerned with drive-response synchronization for a class of fuzzy cellular neural networks with time varying delays. Based on the exponential dichotomy of linear differential equations, the Banach fixed point theorem and the differential inequality technique, we obtain the existence of almost periodic solutions of this class of networks. Then, we design a state feedback and an impulsive controller, and construct a suitable Lyapunov function to study the problem of global exponential almost periodic synchronization for the drive-response systems considered. At the end of the paper, we provide an example to verify the effectiveness of the theoretical results.
\end{abstract}

Keywords: almost periodic solution, fuzzy cellular neural networks, time-varying delays, synchronization, state feedback, impulsive control.

\section{Introduction}

Neural networks have been extensively studied for their potential applications in many engineering and scientific fields, such as image processing, associative memory, computing technology, nonlinear programming, and so on (Arik, 2002b; 2002a; Sen, 2006; Cao and Liand, 2004; Xia et al., 2007; Xu and Yang, 2005). There are many research results, especially for Hopfield neural networks (Aouiti et al., 2017), cellular neural networks (Park, 2009; Pan and Cao, 2011), bidirectional associative memory neural networks (Aouiti et al., 2017) and Cohen-Grossberg neural networks (Yang et al., 2017). However, when using mathematical modeling to solve real-world problems, the uncertainty of vagueness is encountered. In order to solve the problems of vagueness, Yang and Yang (1996) applied fuzzy logic to the traditional cellular neural networks, which integrates fuzzy logic into the structure of traditional cellular neural networks and maintains local connectedness among cells.

Unlike previous cellular neural network structures,

${ }^{*}$ Corresponding author fuzzy cellular neural networks have fuzzy logic between their template and input and/or output besides the "sum of products" operation. Combing the advantages of fuzzy operations and cellular neural networks, fuzzy neural networks have wide applications in image processing and pattern recognition problems. Therefore, there are a lot of results for fuzzy cellular neural networks; see the works of Li and Wang (2013), Yuan et al. (2006), Long and $\mathrm{Xu}$ (2011) or Li and Zhang (2009) and the references therein. Moreover, it is well known that periodicity, anti-periodicity and almost periodicity are very important dynamics for non-autonomous neural networks (Li et al., 2009; Pan and Cao, 2011; Li and Fan, 2009). In addition, in the real world, almost periodicity is more realistic and more general than periodicity. Also, time delays are inevitable both in biological and artificial neural networks. Recently, the almost periodic problem of fuzzy cellular neural networks with time delays were considered by Huang (2017a; 2017b).

Pecora and Carroll (1990) proposed the concept of synchronization. Its mechanism is as follows: a chaotic system, called the driver (or master) system, 
generates a signal and sends it to a response (or slave) system over a channel. The response system is identical with the driver system. Then the response system uses this signal to control itself so that it oscillates in a manner synchronized with the drive system. Since then, due to its wide applications in engineering such as in secure communication, biological systems or information processing, much attention has been attracted to this topic. There are many synchronization strategies, such as coupling control, state feedback control, adaptive control and impulsive control (Heagy et al., 1994; Yang, 2001; Cao et al, 2005; Tang et al., 2017a; 2018b; Yuan et al., 2014). In the past few years, the problem of periodic synchronization for systems has been extensively investigated (see Hu et al., 2010; Hong, 2014; Cai et al., 2015; Wu et al., 2015). Very recently, the problems of almost periodic synchronization, almost automorphic synchronization and pseudo almost periodic synchronization for quaternion-valued neural networks have been studied by Li et al. (2018b; 2018c; 2018a), respectively.

It should be noted that the impulsive control method has found favor with many researchers, since it allows synchronization of systems to use the impulsive controllers with only small impulses, generated by nodes of the state variables of the drive and the response systems at discrete time instants. Moreover, when the synchronization impulses are sent to the receiving systems at discrete time instants, this can drastically decrease information redundancy in the transmitted signal and increase robustness against disturbances, which can greatly reduce control cost. Recently, some interesting synchronization results for chaotic neural networks have been reported using the impulsive control method, see the works of Cao et al. (2009), Lu et al., (2013; 2018), Yang et al. (2015; 2018), Guan (2018), Zhang et al. (2018) or Lin and Zhang (2018) and the references therein.

Up to now, although there have been few studies on synchronization of fuzzy cellular neural networks (Ding and Han, 2008; Abdurahman et al., 2016; Wang, 2018; Ding et al., 2009; Feng et al., 2011; Yang et al., 2017), almost periodic synchronization of fuzzy cellular neural networks via impulsive controls has not been studied. But it is interesting and challenging.

Inspired by the above analysis, in this paper, we are concerned with the following fuzzy cellular neural network with time-varying delays:

$$
\begin{aligned}
x_{i}^{\prime}(t)= & -a_{i}(t) x_{i}(t)+\sum_{j=1}^{n} b_{i j}(t) f_{j}\left(x_{j}(t)\right) \\
& +\sum_{j=1}^{n} c_{i j}(t) g_{j}\left(x_{j}\left(t-\sigma_{i j}(t)\right)\right)
\end{aligned}
$$

$$
\begin{aligned}
& +\sum_{j=1}^{n} d_{i j}(t) \mu_{j}(t)+\bigwedge_{j=1}^{n} T_{i j}(t) \mu_{j}(t) \\
& +\bigwedge_{j=1}^{n} \alpha_{i j}(t) h_{j}\left(x_{j}\left(t-\tau_{i j}(t)\right)\right) \\
& +\bigvee_{j=1}^{n} \beta_{i j}(t) h_{j}\left(x_{j}\left(t-\eta_{i j}(t)\right)\right) \\
& +\bigvee_{j=1}^{n} S_{i j}(t) \mu_{j}(t)+I_{i}(t),
\end{aligned}
$$

where $i \in\{1,2, \ldots, n\}:=\Pi, n$ corresponds to the number of units in a neural network, $x_{i}(t)$ is the state variable of the $i$-th neuron at time $t ; a_{i}(t)>$ 0 represents the rate with which the $i$-th neuron will reset its potential to the resting state in isolation when disconnected from the networks and external inputs; $\alpha_{i j}, \beta_{i j}, T_{i j}, S_{i j}$ are elements of the fuzzy feedback MIN template, fuzzy feedback MAX template, fuzzy feedforward MIN template, fuzzy feedforward MAX template, respectively; $\bigwedge, \bigvee$ denote the fuzzy AND and OR operation, respectively; $b_{i j}, d_{i j}$ are elements of the feedback and free-forward template; $c_{i j}$ denotes the weight strength of the $i$-th neuron at time $t ; \mu_{i}$ denotes an input of the $i$ th neuron; $I_{i}(t)$ denotes the external input to the $i$ th neuron at time $t ; f_{j}, g_{j}, h_{j}: \mathbb{R} \rightarrow \mathbb{R}$ are the activation functions of signal transmission; $\sigma_{i j}(t)>$ $0, \tau_{i j}(t)>0, \eta_{i j}(t)>0$ correspond to transmission delays.

Throughout the paper, for convenience, for a bounded continuous function, we use the notation

$$
f^{+}=\sup _{t \in \mathbb{R}}|f(t)|, \quad f^{-}=\inf _{t \in \mathbb{R}}|f(t)| .
$$

The initial conditions associated with the system (1) are of the form

$$
x_{i}(s)=\varphi_{i}(s), \quad s \in[-\theta, 0], \quad i \in \Pi,
$$

where

$$
\begin{aligned}
& \theta=\max \{\sigma, \tau, \eta\}, \quad \sigma=\max _{1 \leq i, j \leq n}\left\{\sigma_{i j}^{+}\right\}, \\
& \tau=\max _{1 \leq i, j \leq n}\left\{\tau_{i j}^{+}\right\}, \quad \eta=\max _{1 \leq i, j \leq n}\left\{\eta_{i j}^{+}\right\},
\end{aligned}
$$

and $\varphi_{i}(\cdot)$ is a real- valued bounded continuous function defined on $[-\theta, 0]$.

The main purpose of this paper is to study the problem of almost periodic synchronization of (1) via state-feedback and impulsive control. To the best of our knowledge, this is the first paper to study almost periodic synchronization for fuzzy cellular neural networks. Our results are completely new and our methods can be used to study the problem of almost periodic and almost automorphic synchronization for other types of neural networks. 
Remark 1. Following ideas similar to those used by Cai et al. (2015), Hu et al. (2010) or $\mathrm{Li}$ et al. (2018b; 2018a; 2018c), in this paper, drive-response synchronization of systems with almost periodic coefficients and almost periodic solutions is called almost periodic synchronization.

We organize the paper as follows. In Section 2, we introduce the notation and definitions, and state some preliminary results which are needed in further sections. In Section 3, sufficient conditions for the existence of almost periodic solutions of system (1) are obtained. In Section 4, the exponential synchronization is investigated. In Section 5, the effectiveness and feasibility of the developed methods are shown with a numerical example. The paper ends with a brief conclusion in Section 6.

\section{Preliminaries}

In this section, we shall recall some fundamental definitions and lemmas.

Definition 1. (Fink, 1974) Let $f \in B C\left(\mathbb{R}, \mathbb{R}^{n}\right)$; then $f$ is said to be almost periodic if, for any $\epsilon>0$, it is possible to find a real number $l=l(\epsilon)>0$, such that for any interval of length $l(\epsilon)$, there exists a number $\tau=\tau(\epsilon)$ in this interval satisfying $|f(t+\tau)-f(t)|<\epsilon$ for all $t \in \mathbb{R}$.

We denote by $A P\left(\mathbb{R}, \mathbb{R}^{n}\right)$ the set of all almost periodic functions from $\mathbb{R}$ to $\mathbb{R}^{n}$.

Lemma 1. (Diagana, 2013) If $f, g \in A P\left(\mathbb{R}, \mathbb{R}^{n}\right)$, then $f+g, f g \in A P\left(\mathbb{R}, \mathbb{R}^{n}\right)$.

Consider the following linear homogenous system:

$$
x^{\prime}(t)=A(t) x(t)
$$

and the linear non-homogenous system

$$
x^{\prime}(t)=A(t) x(t)+f(t)
$$

where $A(t)$ is an almost periodic matrix function and $f(t)$ is an almost periodic vector function.

Definition 2. (Fink, 1974) The system (2) is said to admit an exponential dichotomy if there exist a projection $P$ and positive constants $\alpha, \beta$ such that the fundamental solution matrix $X(t)$ satisfies

$$
\begin{aligned}
\left|X(t) P X^{-1}(s)\right| & \leq \beta e^{-\alpha(t-s)}, & & t \geq s, \\
\left|X(t)(I-P) X^{-1}(s)\right| & \leq \beta e^{-\alpha(s-t)}, & & t \leq s .
\end{aligned}
$$

Lemma 2. (Fink, 1974) If the linear system (2) admits an exponential dichotomy, then the system (3) has a unique almost periodic solution

$$
x(t)=\int_{-\infty}^{t} X(t) P X^{-1}(s) f(s) \mathrm{d} s
$$

$$
-\int_{t}^{+\infty} X(t)(I-P) X^{-1}(s) f(s) \mathrm{d} s,
$$

where $X(t)$ is the fundamental solution matrix of (2).

Lemma 3. (Fink, 1974) Let $a_{i}$ be an almost periodic function on $\mathbb{R}$ and

$$
M\left[a_{i}\right]:=\lim _{T \rightarrow \infty} \frac{1}{T} \int_{t}^{t+T} a_{i}(s) \mathrm{d} s>0, \quad i \in \Pi .
$$

Then the linear system

$$
x^{\prime}(t)=\operatorname{diag}\left(-a_{1}(t),-a_{2}(t), \ldots,-a_{n}(t)\right) x(t)
$$

admits an exponential dichotomy on $\mathbb{R}$.

Lemma 4. (Yang nad Yang, 1996) Let $x_{j}, y_{j}, \alpha_{i j}, \beta_{i j} \in$ $\mathbb{R}, f_{j}: \mathbb{R} \rightarrow \mathbb{R}$ be continuous functions where $i, j \in \Pi$; then we have

$$
\begin{aligned}
& \left|\bigwedge_{j=1}^{n} \alpha_{i j} f_{j}\left(x_{j}\right)-\bigwedge_{j=1}^{n} \alpha_{i j} f_{j}\left(y_{j}\right)\right| \\
& \quad \leq \sum_{j=}^{n}\left|\alpha_{i j}\right|\left|f_{j}\left(x_{j}\right)-f_{j}\left(y_{j}\right)\right| \\
& \left|\bigvee_{j=1}^{n} \beta_{i j} f_{j}\left(x_{j}\right)-\bigvee_{j=1}^{n} \beta_{i j} f_{j}\left(y_{j}\right)\right| \\
& \quad \leq \sum_{j=}^{n}\left|\beta_{i j}\right|\left|f_{j}\left(x_{j}\right)-f_{j}\left(y_{j}\right)\right|
\end{aligned}
$$

Similarly to the proof by Huang (2017b, Remark 2.2), we have the following result.

Lemma 5. For $i, j \in \Pi$, let $x_{j}, \mu_{j}, \tau_{i j}, \eta_{i j}, \alpha_{i j}$, $\beta_{i j}, T_{i j}, S_{i j} \in A P(\mathbb{R}, \mathbb{R})$ and $\left(A_{2}\right)$ hold; then we have $\bigwedge_{j=1}^{n} \alpha_{i j}(\cdot) h_{j}\left(x_{j}\left(\cdot-\tau_{i j}(\cdot)\right)\right), \bigvee_{j=1}^{n} \beta_{i j}(\cdot) h_{j}\left(x_{j}(\cdot-\right.$ $\left.\left.\eta_{i j}(\cdot)\right)\right), \bigwedge_{j=1}^{n} T_{i j}(\cdot) \mu_{j}, \bigvee_{j=1}^{n} S_{i j}(\cdot) \mu_{j} \in A P(\mathbb{R}, \mathbb{R})$.

Throughout the paper, we make the following assumptions:

$\left(A_{1}\right)$ Function $a_{i} \in A P\left(\mathbb{R}, \mathbb{R}^{+}\right)$with $M\left[a_{i}\right]>0$, $b_{i j}, c_{i j}, d_{i j}, \alpha_{i j}, \beta_{i j}, S_{i j}, T_{i j}, \mu_{i}, I_{i} \in A P(\mathbb{R}, \mathbb{R})$, $\sigma_{i j}, \tau_{i j}, \eta_{i j} \in C^{1}\left(\mathbb{R}, \mathbb{R}^{+}\right) \cap A P(\mathbb{R}, \mathbb{R})$ with

$$
\begin{aligned}
& \gamma=\inf _{t \in \mathbb{R}}\left\{1-\sigma_{i j}^{\prime}(t)\right\}>0, \\
& \rho=\inf _{t \in \mathbb{R}}\left\{1-\tau_{i j}^{\prime}(t)\right\}>0, \\
& \varrho=\inf _{t \in \mathbb{R}}\left\{1-\eta_{i j}^{\prime}(t)\right\}>0,
\end{aligned}
$$

where $i, j \in \Pi$.

$\left(A_{2}\right)$ For any $u, v \in \mathbb{R}$, there exist positive constants $L_{j}^{f}, L_{j}^{g}, L_{j}^{h}$ such that

$$
\left|f_{j}(u)-f_{j}(v)\right| \leq L_{j}^{f}|u-v|,
$$




$$
\begin{aligned}
\left|g_{j}(u)-g_{j}(v)\right| & \leq L_{j}^{g}|u-v|, \\
\left|h_{j}(u)-h_{j}(v)\right| & \leq L_{j}^{h}|u-v|
\end{aligned}
$$

and $f_{j}(0)=g_{j}(0)=h_{j}(0)=0$, where $j \in \Pi$.

$\left(A_{3}\right)$ There exists a positive constant $r$ such that

$$
\max _{i \in J}\left\{\frac{P_{i}}{a_{i}^{-}}\right\}:=r<1,
$$

where $i \in \Pi$ and

$$
P_{i}=\sum_{j=1}^{n}\left[b_{i j}^{+} L_{j}^{f}+c_{i j}^{+} L_{j}^{g}+\alpha_{i j}^{+} L_{j}^{h}+\beta_{i j}^{+} L_{j}^{h}\right] .
$$

\section{Existence of almost periodic solutions}

In this section, we shall state and prove sufficient conditions for the existence and uniqueness of almost periodic solutions of the system (11).

Set $\mathbb{Y}=\left\{\varphi=\left(\varphi_{1}, \varphi_{2}, \ldots \varphi_{n}\right)^{T}: \varphi_{i} \in\right.$ $A P(\mathbb{R}, \mathbb{R}), i \in \Pi\}$ and equip it with the norm $\|\varphi\|=$ $\max _{i \in \Pi}\left\{\sup _{t \in \mathbb{R}}\left|\varphi_{i}(t)\right|\right\}$. Then $\mathbb{Y}$ is a Banach space. Let

$$
\varphi^{0}(t)=\left(\varphi_{1}^{0}(t), \varphi_{2}^{0}(t), \ldots, \varphi_{n}^{0}(t)\right)^{T}
$$

where

$$
\begin{aligned}
\varphi_{i}^{0}(t)= & \int_{-\infty}^{t} e^{-\int_{s}^{t} a_{i}(u) \mathrm{d} u}\left[\sum_{j=1}^{n} d_{i j}(s) \mu_{j}(s)\right. \\
& \left.+\bigwedge_{j=1}^{n} T_{i j}(s) \mu_{j}(s)+\bigvee_{j=1}^{n} S_{i j}(s) \mu_{j}(s)+I_{i}(s)\right] \mathrm{d} s
\end{aligned}
$$

$i \in \Pi$ and $L$ be a constant satisfying $L \geq\left\|\varphi^{0}\right\|$.

Theorem 1. Let $\left(A_{1}\right)-\left(A_{3}\right)$ hold. Then the system (1) has a unique almost periodic solution in $\mathbb{Y}^{*}=\{\varphi \in \mathbb{Y} \mid \| \varphi-$ $\left.\varphi^{0} \| \leq r L /(1-r)\right\}$.

Proof. For any given $\varphi \in \mathbb{Y}$, consider the following linear system:

$$
x_{i}^{\prime}(t)=-a_{i}(t) x_{i}(t)+F_{i}(t, \varphi(t))+E_{i}(t), \quad i \in \Pi,
$$

where

$$
\begin{aligned}
F_{i}(t, \varphi(t))= & \sum_{j=1}^{n} b_{i j}(t) f_{j}\left(\varphi_{j}(t)\right)+\sum_{j=1}^{n} c_{i j}(t) \\
& \times g_{j}\left(\left(\varphi_{j}\left(t-\sigma_{i j}(t)\right)\right)\right. \\
& +\bigwedge_{j=1}^{n} \alpha_{i j}(t) h_{j}\left(\varphi_{j}\left(t-\tau_{i j}(t)\right)\right) \\
& +\bigvee_{j=1}^{n} \beta_{i j}(t) h_{j}\left(\varphi_{j}\left(t-\eta_{i j}(t)\right)\right),
\end{aligned}
$$

$$
\begin{aligned}
E_{i}(t)= & \sum_{j=1}^{n} d_{i j}(t) \mu_{j}(t)+\bigwedge_{j=1}^{n} T_{i j}(t) \mu_{j}(t) \\
& +\bigvee_{j=1}^{n} S_{i j}(t) \mu_{j}(t)+I_{i}(t) .
\end{aligned}
$$

From Assumption $A_{1}$ and Lemma 3 it follows that the linear system

$$
x_{i}^{\prime}(t)=-a_{i}(t) x_{i}(t), \quad i \in \Pi,
$$

admits an exponential dichotomy. By Lemma 2, we have that the system (4) has a unique almost periodic solution $x^{\varphi}=\left(x_{1}^{\varphi}, x_{2}^{\varphi}, \ldots x_{n}^{\varphi}\right)$, where

$$
\begin{aligned}
x_{i}^{\varphi}(t)= & \int_{-\infty}^{t} e^{-\int_{s}^{t} a_{i}(u) \mathrm{d} u}\left[F_{i}(s, \varphi(s))\right. \\
& \left.+E_{i}(s)\right] \mathrm{d} s, \quad i \in \Pi .
\end{aligned}
$$

For every $\varphi \in \mathbb{Y}^{*}$, we find

$$
\|\varphi\| \leq\left\|\varphi^{0}\right\|+\left\|\varphi-\varphi^{0}\right\| \leq L+\frac{r L}{1-r}=\frac{L}{1-r} .
$$

Now we define a mapping $\Phi: \mathbb{Y}^{*} \rightarrow \mathbb{Y}^{*}$ by setting $\Phi \varphi=$ $x^{\varphi}, \varphi \in \mathbb{Y}^{*}$.

First, we will prove that, for any $\varphi \in \mathbb{Y}^{*}, \Phi \varphi \in \mathbb{Y}^{*}$. In fact, we have

$$
\begin{aligned}
\mid & F_{i}(s, \varphi(s)) \mid \\
= & \mid \sum_{j=1}^{n} b_{i j}(s) f_{j}\left(\varphi_{j}(s)\right)+\sum_{j=1}^{n} c_{i j}(s) \\
& \times g_{j}\left(\varphi_{j}\left(s-\sigma_{i j}(s)\right)\right) \\
& +\bigwedge_{j=1}^{n} \alpha_{i j}(s) h_{j}\left(\varphi_{j}\left(s-\tau_{i j}(s)\right)\right) \\
& +\bigvee_{j=1}^{n} \beta_{i j}(s) h_{j}\left(\varphi_{j}\left(s-\eta_{i j}(s)\right)\right) \mid \\
\leq & \sum_{j=1}^{n} b_{i j}^{+}\left|f_{j}\left(\varphi_{j}(s)\right)-f_{j}(0)\right| \\
& +\sum_{j=1}^{n} c_{i j}^{+}\left|g_{j}\left(\varphi_{j}\left(s-\sigma_{i j}(s)\right)\right)-g_{j}(0)\right| \\
& +\sum_{j=1}^{n} \alpha_{i j}^{+}\left|h_{j}\left(\varphi_{j}\left(s-\tau_{i j}(s)\right)\right)-h_{j}(0)\right| \\
& +\sum_{j=1}^{n} \beta_{i j}^{+}\left|h_{j}\left(\varphi_{j}\left(s-\eta_{i j}(s)\right)\right)-h_{j}(0)\right| \\
\leq & \left.\sum_{j=1}^{n} b_{i j}^{+} L_{j}^{f} \mid \varphi_{j}(s)\right)\left|+\sum_{j=1}^{n} c_{i j}^{+} L_{j}^{g}\right| \varphi_{j}\left(s-\sigma_{i j}(s)\right) \mid \\
& +\sum_{j=1}^{n} \alpha_{i j}^{+} L_{j}^{h}\left|\varphi_{j}\left(s-\tau_{i j}(s)\right)\right|
\end{aligned}
$$




$$
+\sum_{j=1}^{n} \beta_{i j}^{+} L_{j}^{h}\left|\varphi_{j}\left(s-\eta_{i j}(s)\right)\right|, \quad i \in \Pi .
$$

From this and the definition of $\Phi$ it follows that

$$
\begin{aligned}
& \sup _{t \in \mathbb{R}}\left|(\Phi \varphi)_{i}(t)-\varphi_{i}^{0}(t)\right| \\
& =\sup _{t \in \mathbb{R}}\left|\left\{\int_{-\infty}^{t} e^{-\int_{s}^{t} a_{i}(u) \mathrm{d} u} F_{i}(s, \varphi(s))\right\} \mathrm{d} s\right| \\
& \leq \frac{1}{a_{i}^{-}} \sum_{j=1}^{n}\left[b_{i j}^{+} L_{j}^{f}+c_{i j}^{+} L_{j}^{g}+\alpha_{i j}^{+} L_{j}^{h}+\beta_{i j}^{+} L_{j}^{h}\right]\|\varphi\| \\
& \leq r\|\varphi\| \leq \frac{r L}{1-r}, \quad i \in \Pi,
\end{aligned}
$$

which implies $\Phi \varphi \in \mathbb{Y}^{*}$. Thus, the mapping $\Phi$ is a selfmapping from $\mathbb{Y}^{*}$ to $\mathbb{Y}^{*}$.

Next, we will show that $\Phi$ is a contraction mapping in $\mathbb{Y}^{*}$. For any $\varphi, \psi \in \mathbb{Y}^{*}$, we have

$$
\begin{aligned}
& \left|F_{i}(s, \varphi(s))-F_{i}(s, \psi(s))\right| \\
& \leq \sum_{j=1}^{n} b_{i j}^{+}\left|f_{j}\left(\varphi_{j}(s)\right)-f_{j}\left(\psi_{j}(s)\right)\right| \\
& +\sum_{j=1}^{n} c_{i j}^{+} \mid g_{j}\left(\varphi_{j}\left(s-\sigma_{i j}(s)\right)\right) \\
& -g_{j}\left(\psi_{j}\left(s-\sigma_{i j}(s)\right)\right)\left|+\sum_{j=1}^{n} \alpha_{i j}^{+}\right| h_{j}\left(\varphi_{j}(s\right. \\
& \left.\left.-\tau_{i j}(s)\right)\right)-h_{j}\left(\psi_{j}\left(s-\tau_{i j}(s)\right)\right) \mid \\
& +\sum_{j=1}^{n} \beta_{i j}^{+} \mid h_{j}\left(\varphi_{j}\left(s-\eta_{i j}(s)\right)\right) \\
& -h_{j}\left(\psi_{j}\left(s-\eta_{i j}(s)\right)\right) \mid \\
& \left.\left.\leq \sum_{j=1}^{n} b_{i j}^{+} L_{j}^{f} \mid \varphi_{j}(s)\right)-\psi_{j}(s)\right) \mid \\
& +\sum_{j=1}^{n} c_{i j}^{+} L_{j}^{g}\left|\varphi_{j}\left(s-\sigma_{i j}(s)\right)-\psi_{j}\left(s-\sigma_{i j}(s)\right)\right| \\
& +\sum_{j=1}^{n} \alpha_{i j}^{+} L_{j}^{h}\left|\varphi_{j}\left(s-\tau_{i j}(s)\right)-\psi_{j}\left(s-\tau_{i j}(s)\right)\right| \\
& +\sum_{j=1}^{n} \beta_{i j}^{+} L_{j}^{h} \mid \varphi_{j}\left(s-\eta_{i j}(s)\right) \\
& -\psi_{j}\left(s-\eta_{i j}(s)\right) \mid, \quad i \in \Pi .
\end{aligned}
$$

From the above inequality and the definition of $\Phi$, we obtain

$$
\begin{aligned}
& \sup _{t \in \mathbb{R}}\left|(\Phi \varphi)_{i}(t)-\varphi_{i}^{0}(t)(\Phi \psi)_{i}(t)\right| \\
& \leq \sup _{t \in \mathbb{R}}\left\{\int _ { - \infty } ^ { t } e ^ { - \int _ { s } ^ { t } a _ { i } ( u ) \mathrm { d } u } \sum _ { j = 1 } ^ { n } \left[b_{i j}^{+} L_{j}^{f}+c_{i j}^{+} L_{j}^{g}\right.\right.
\end{aligned}
$$

$$
\begin{aligned}
& \left.\left.+\alpha_{i j}^{+} L_{j}^{h}+\beta_{i j}^{+} L_{j}^{h}\right]\|\varphi-\psi\| \mathrm{d} s\right\} \\
\leq & \frac{P_{i}}{a_{i}^{-}}\|\varphi-\psi\|, \quad i \in \Pi .
\end{aligned}
$$

Therefore, we get

$$
\|\Phi \varphi-\Phi \psi\| \leq r\|\varphi-\psi\| .
$$

By Assumption $A_{3}, \Phi$ is a contraction mapping. Based on the Banach fixed theorem, we obtain that $\Phi$ has a unique fixed point in $\Omega$, which means that the system (1) has a unique almost periodic solution in $\mathbb{Y}^{*}$. The proof is complete.

\section{Almost periodic synchronization via state-feedback and impulsive control}

In this section, by utilizing some analytic techniques and constructing a suitable Lyapunov function, we will investigate the exponential synchronization problem of fuzzy cellular neural networks with time-varying delays and almost periodic coefficients. For this purpose, we consider the system (1) as the drive system, and the response system is designed as

$$
\begin{aligned}
y_{i}^{\prime}(t)= & -a_{i}(t) y_{i}(t)+\sum_{j=1}^{n} b_{i j}(t) f_{j}\left(y_{j}(t)\right) \\
& +\sum_{j=1}^{n} c_{i j}(t) g_{j}\left(y_{j}\left(t-\sigma_{i j}(t)\right)\right) \\
& +\sum_{j=1}^{n} d_{i j}(t) \mu_{j}(t)+\bigwedge_{j=1}^{n} T_{i j}(t) \mu_{j}(t) \\
& +\bigwedge_{j=1}^{n} \alpha_{i j}(t) h_{j}\left(y_{j}\left(t-\tau_{i j}(t)\right)\right) \\
& +\bigvee_{j=1}^{n} \beta_{i j}(t) h_{j}\left(y_{j}\left(t-\eta_{i j}(t)\right)\right) \\
& +\bigvee_{j=1}^{n} S_{i j}(t) \mu_{j}(t)+I_{i}(t) \\
& +u_{i}(t), \quad i \in \Pi,
\end{aligned}
$$

where $u_{i}(t)$ is the control input to be designed. The initial condition of (5) is as follows:

$$
y_{i}(s)=\psi_{i}(s), \quad s \in[-\nu, 0], \quad i \in \Pi,
$$

where $\nu=\max \{\sigma, \tau, \eta, \xi\}, \xi=\max _{1 \leq i, j \leq n}\left\{\xi_{i j}^{+}\right\}$, and $\psi_{i}(\cdot)$ is a real-valued continuous function defined on $[-\nu, 0]$.

Let $z_{i}(t)=y_{i}(t)-x_{i}(t)$ be the synchronization error; then we can obtain the following error dynamical 
system of (1) and (5):

$$
\begin{aligned}
z_{i}^{\prime}(t)= & -a_{i}(t) z_{i}(t)+\sum_{j=1}^{n} b_{i j}(t) F_{j}\left(z_{j}(t)\right) \\
& +\sum_{j=1}^{n} c_{i j}(t) G_{j}\left(z_{j}\left(t-\sigma_{i j}(t)\right)\right) \\
& +\bigwedge_{j=1}^{n} \alpha_{i j}(t) H_{j}\left(z_{j}\left(t-\tau_{i j}(t)\right)\right) \\
& +\bigvee_{j=1}^{n} \beta_{i j}(t) \hat{H}_{j}\left(z_{j}\left(t-\eta_{i j}(t)\right)\right) \\
& +u_{i}(t), \quad i \in \Pi,
\end{aligned}
$$

where $F_{j}\left(z_{j}(t)\right) \triangleq f_{j}\left(y_{j}(t)\right)-f_{j}\left(x_{j}(t)\right), G_{j}\left(z_{j}(t-\right.$ $\left.\left.\sigma_{i j}(t)\right)\right) \triangleq g_{j}\left(y_{j}\left(t-\sigma_{i j}(t)\right)\right)-g_{j}\left(x_{j}\left(t-\sigma_{i j}(t)\right)\right)$, $H_{j}\left(z_{j}\left(t-\tau_{i j}(t)\right)\right) \triangleq h_{j}\left(y_{j}\left(t-\tau_{i j}(t)\right)\right)-h_{j}\left(x_{j}(t-\right.$ $\left.\left.\tau_{i j}(t)\right)\right), \hat{H}_{j}\left(z_{j}\left(t-\eta_{i j}(t)\right)\right) \triangleq h_{j}\left(y_{j}\left(t-\eta_{i j}(t)\right)\right)-$ $h_{j}\left(x_{j}\left(t-\eta_{i j}(t)\right)\right)$.

The impulsive controller $u_{i}(t)$ is designed as

$$
\begin{aligned}
u_{i}(t)= & \sum_{k=1}^{\infty} J_{i k}\left(z_{i}(t)\right) \delta\left(t-t_{k}\right)-\kappa_{i}(t) z_{i}(t) \\
& +\sum_{j=1}^{n} e_{i j}(t) l_{j}\left(z_{j}\left(t-\xi_{i j}(t)\right)\right), \quad i \in \Pi,
\end{aligned}
$$

where $k \in \mathbb{Z}^{+}, \delta(\cdot)$ is the Dirac impulsive function.

With the impulsive controller (7), similarly to the proof by Zhang et al. (2018) and using the property of $\delta(\cdot)$, the error system (5) becomes the following

$$
\left\{\begin{aligned}
z_{i}^{\prime}(t)=- & a_{i}(t) z_{i}(t)+\sum_{j=1}^{n} b_{i j}(t) F_{j}\left(z_{j}(t)\right) \\
& +\sum_{j=1}^{n} c_{i j}(t) G_{j}\left(z_{j}\left(t-\sigma_{i j}(t)\right)\right) \\
& +\bigwedge_{j=1}^{n} \alpha_{i j}(t) H_{j}\left(z_{j}\left(t-\tau_{i j}(t)\right)\right) \\
& +\bigvee_{j=1}^{n} \beta_{i j}(t) \hat{H}_{j}\left(z_{j}\left(t-\eta_{i j}(t)\right)\right) \\
& -\kappa_{i}(t) z_{i}(t)+\sum_{j=1}^{n} e_{i j}(t) \\
& \times l_{j}\left(z_{j}\left(t-\xi_{i j}(t)\right)\right), \quad t \neq t_{k}, \\
& =z_{i k}\left(z_{i}\left(t_{k}^{-}\right)\right), \quad t=t_{k}, \\
\triangle z_{i}\left(t_{k}\right) & \quad s \in[-\nu, 0], \\
z_{i}(s)= & \psi_{i}(s)-\varphi_{i}(s), \quad s \in
\end{aligned}\right.
$$

where $i \in \Pi, k \in \mathbb{Z}^{+}, \Delta z_{i}\left(t_{k}\right)$ denotes the state jumping at impulsive time instant $t=t_{k}$, while $z_{i}\left(t_{k}^{+}\right)$and $z_{i}\left(t_{k}^{-}\right)$ are the right-hand and left-hand limits of function $z_{i}(t)$ at $t_{k}$. Moreover, $z_{i}\left(t_{k}^{+}\right)=z_{i}\left(t_{k}\right)$ and

$$
J_{k}\left(z_{i}\left(t_{k}^{-}\right)\right)
$$

$$
=\left(J_{1 k}\left(z_{1}\left(t_{k}^{-}\right)\right), J_{2 k}\left(z_{2}\left(t_{k}^{-}\right)\right), \ldots, J_{n k}\left(z_{n}\left(t_{k}^{-}\right)\right)\right)^{T}
$$

represents impulsive perturbation of the $i$-th neuron at $t_{k}$.

Set $\mathcal{B}=\left\{\left\{t_{k}\right\}: t_{k} \in \mathbb{R}, t_{k}<t_{k+1}, k \in\right.$ $\left.\mathbb{Z}^{+}, \lim _{k \rightarrow \pm \infty} t_{k}= \pm \infty\right\}$. For $\left\{t_{k}\right\} \in \mathcal{B}$, let $P C\left(\mathbb{R}, \mathbb{R}^{n}\right)$ be the space formed by all bounded piecewise continuous functions $\varphi: \mathbb{R} \rightarrow \mathbb{R}^{n}$ such that $\varphi(\cdot)$ is continuous at $t$ for any $t \notin\left\{t_{k}\right\}$ and $\varphi\left(t_{k}\right)=\varphi\left(t_{k}^{+}\right)$for all $k \in \mathbb{Z}^{+}$.

Definition 3. (Stamov, 2012) The function $\varphi \in$ $P C\left(\mathbb{R}, \mathbb{R}^{n}\right)$ is said to be almost periodic if the following holds:

(i) $\left\{t_{k}\right\}$ constitutes a uniformly almost periodic sequence.

(ii) For any $\epsilon>0$, there exists a positive number $\delta=$ $\delta(\epsilon)$ such that, if the points $t^{\prime}$ and $t^{\prime \prime}$ belong to the same interval of continuity of $\varphi(t)$ and $\left|t^{\prime}-t^{\prime \prime}\right|<\delta$, then $\left\|\varphi\left(t^{\prime}\right)-\varphi\left(t^{\prime \prime}\right)\right\|<\epsilon$.

(iii) For every $\epsilon>0$, there exists a relatively dense set $T$ such that, if $\tau \in T$, then $\|\varphi(t+\tau)-\varphi(t)\|<\epsilon$ for all $t \in \mathbb{R}$ satisfying the condition $\left|t-t_{k}\right|>\epsilon, k \in \mathbb{Z}^{+}$.

Definition 4. The response system (5) and the drive system (11) are said to be globally exponentially synchronized if there exist positive constants $M>0$ and $\lambda>0$ such that

$$
\|y(t)-x(t)\| \leq M\|\psi-\varphi\|_{0} e^{-\lambda t},
$$

where

$$
\begin{gathered}
\|y(t)-x(t)\|=\max _{i \in \Pi}\left\{\left|y_{i}(t)-x_{i}(t)\right|\right\}, \\
\|\psi-\varphi\|_{0}=\max _{i \in \Pi}\left\{\sup _{t \in[-\nu, 0]}\left|\psi_{i}(t)-\varphi_{i}(t)\right|\right\} .
\end{gathered}
$$

Theorem 2. Let $\left(A_{1}\right)-\left(A_{3}\right)$ hold and suppose further the following:

$\left(A_{4}\right) \kappa_{i} \in P C\left(\mathbb{R}, \mathbb{R}^{+}\right), e_{i j} \in P C(\mathbb{R}, \mathbb{R})$ and $\xi_{i j} \in$ $P C^{1}\left(\mathbb{R}, \mathbb{R}^{+}\right)$with $\varsigma=\inf _{t \in \mathbb{R}}\left\{1-\xi_{i j}^{\prime}(t)\right\}>0$ are almost periodic, where $i, j \in \Pi$.

$\left(A_{5}\right)$ For any $u, v \in \mathbb{R}$, there exists a positive constant $L_{j}^{l}$ such that

$$
\left|l_{j}(u)-l_{j}(v)\right| \leq L_{l}^{l}|u-v|
$$

and $l_{j}(0)=0$, where $j \in \Pi$.

$\left(A_{6}\right)$ The impulsive operator $J_{i k}\left(z_{i}\left(t_{k}^{-}\right)\right)$satisfies $\left.J_{i k}\left(z_{i}\left(t_{k}^{-}\right)\right)=-\lambda_{i k}\left(z_{i}\left(t_{k}^{-}\right)\right)\right), 0<\lambda_{i k}<2, i \in \Pi$, $k \in \mathbb{Z}^{+}$. 
$\left(A_{7}\right)$ There exists a positive constant $\omega$ such that

$$
\begin{aligned}
\max _{i \in \Pi}\left\{\omega-\left(a_{i}^{-}+\kappa_{i}^{-}\right)+\sum_{j=1}^{n} b_{i j}^{+} L_{j}^{f}\right. \\
+\frac{e^{\omega \sigma}}{\gamma} \sum_{j=1}^{n} c_{i j}^{+} L_{j}^{g}+\frac{e^{\omega \tau}}{\rho} \sum_{j=1}^{n} \alpha_{i j}^{+} L_{j}^{h} \\
\left.+\frac{e^{\omega \eta}}{\varrho} \sum_{j=1}^{n} \beta_{i j}^{+} L_{j}^{h}+\frac{e^{\omega \xi}}{\varsigma} \sum_{j=1}^{n} e_{i j}^{+} L_{j}^{l}\right\}<0 .
\end{aligned}
$$

Then the drive system (1) and the response system (5) are globally exponentially synchronized.

Proof. For $t>0$, and $t \neq t_{k}, k \in \mathbb{Z}^{+}$, we have

$$
\begin{aligned}
& \left|z_{i}^{\prime}(t)\right|=\left|y_{i}^{\prime}(t)-x_{i}^{\prime}(t)\right| \\
& =\mid-a_{i}(t)\left[y_{i}(t)-x_{i}(t)\right]+\sum_{j=1}^{n} b_{i j}(t) \\
& \times\left[f_{j}\left(y_{j}(t)\right)-f_{j}\left(x_{j}(t)\right)\right] \\
& +\sum_{j=1}^{n} c_{i j}(t)\left[g_{j}\left(y_{j}\left(t-\sigma_{i j}(t)\right)\right)\right. \\
& \left.-g_{j}\left(x_{j}\left(t-\sigma_{i j}(t)\right)\right)\right] \\
& +\bigwedge_{j=1}^{n} \alpha_{i j}(t)\left[h_{j}\left(y_{j}\left(t-\tau_{i j}(t)\right)\right)\right. \\
& \left.-h_{j}\left(x_{j}\left(t-\tau_{i j}(t)\right)\right)\right] \\
& +\bigvee_{j=1}^{n} \beta_{i j}(t)\left[h_{j}\left(y_{j}\left(t-\eta_{i j}(t)\right)\right)\right. \\
& \left.-h_{j}\left(x_{j}\left(t-\eta_{i j}(t)\right)\right)\right]-\kappa_{i}(t) z_{i}(t) \\
& +\sum_{j=1}^{n} e_{i j}(t) l_{j}\left(z_{j}\left(t-\xi_{i j}(t)\right)\right) \\
& \leq-\left(a_{i}^{-}+\kappa_{i}^{-}\right)\left|z_{i}(t)\right|+\sum_{j=1}^{n} b_{i j}^{+} L_{j}^{f}\left|z_{j}(t)\right| \\
& \left.+\sum_{j=1}^{n} c_{i j}^{+} L_{j}^{g} \mid z_{j}\left(t-\sigma_{i j}(t)\right)\right) \mid \\
& +\sum_{j=1}^{n} \alpha_{i j}^{+} L_{j}^{h}\left|z_{j}\left(t-\tau_{i j}(t)\right)\right| \\
& +\sum_{j=1}^{n} \beta_{i j}^{+} L_{j}^{h}\left|z_{j}\left(t-\eta_{i j}(t)\right)\right| \\
& +\sum_{j=1}^{n} e_{i j}^{+} L_{j}^{l}\left|z_{j}\left(t-\xi_{i j}(t)\right)\right|, \quad i \in \Pi .
\end{aligned}
$$

Also, for $t=t_{k}, k \in \mathbb{Z}^{+}$, we obtain

$$
z_{i}\left(t_{k}^{+}\right)=z_{i}\left(t_{k}^{-}\right)-\lambda_{i k}\left(z_{i}\left(t_{k}^{-}\right)\right)
$$

$$
=\left(1-\lambda_{i k}\right)\left(z_{i}\left(t_{k}^{-}\right)\right), \quad i \in \Pi \text {. }
$$

Then, by $\left(A_{6}\right)$ and (9), we obtain

$$
\left|z_{i}\left(t_{k}^{+}\right)\right| \leq\left|z_{i}\left(t_{k}^{-}\right)\right|, \quad k \in \mathbb{Z}^{+}, \quad i \in \Pi .
$$

With $W_{i}(t)=e^{\omega t}\left|z_{i}(t)\right|$, for $t \neq t_{k}$, we have

$$
W_{i}^{\prime}(t) \leq \omega W_{i}(t)+e^{\omega t}\left|z_{i}^{\prime}(t)\right|, \quad i \in \Pi
$$

and for $t=t_{k}$, we have

$$
\begin{aligned}
W_{i}\left(t_{k}^{+}\right) & =e^{\omega t_{k}^{+}}\left|z_{i}\left(t_{k}^{+}\right)\right| \leq e^{\omega t_{k}^{-}}\left|z_{i}\left(t_{k}^{-}\right)\right| \\
& =W_{i}\left(t_{k}^{-}\right), \quad k \in \mathbb{Z}^{+}, \quad i \in \Pi .
\end{aligned}
$$

Let us construct a Lyapunov function $V(t)$ as follows:

$$
V(t)=\sum_{i=1}^{n}\left\{W_{i}(t)+\Lambda_{i}(t)\right\},
$$

where $i \in \Pi$ and

$$
\begin{aligned}
\Lambda_{i}(t)= & \frac{e^{\omega \sigma}}{\gamma} \sum_{j=1}^{n} c_{i j}^{+} L_{j}^{g} \int_{t-\sigma_{i j}(t)}^{t}\left|z_{j}(s)\right| e^{\omega s} \mathrm{~d} s \\
& +\frac{e^{\omega \tau}}{\rho} \sum_{j=1}^{n} \alpha_{i j}^{+} L_{j}^{h} \int_{t-\tau_{i j}(t)}^{t}\left|z_{j}(s)\right| e^{\omega s} \mathrm{~d} s \\
& +\frac{e^{\omega \eta}}{\varrho} \sum_{j=1}^{n} \beta_{i j}^{+} L_{j}^{h} \int_{t-\eta_{i j}(t)}^{t}\left|z_{j}(s)\right| e^{\omega s} \mathrm{~d} s \\
& +\frac{e^{\omega \xi}}{\varsigma} \sum_{j=1}^{n} e_{i j}^{+} L_{j}^{l} \int_{t-\xi_{i j}(t)}^{t}\left|z_{j}(s)\right| e^{\omega s} \mathrm{~d} s .
\end{aligned}
$$

For $t \neq t_{k}$, calculating the Dini right-hand derivative of $V(t)$ along the solutions of the error system $[6$, we obtain

$$
\begin{aligned}
& D^{+} V(t) \\
& \leq \sum_{i=1}^{n}\left\{\omega W_{i}(t)+e^{\omega t}\left|z_{i}^{\prime}(t)\right|+\frac{e^{\omega \sigma}}{\gamma} \sum_{j=1}^{n} c_{i j}^{+}\right. \\
& \quad \times L_{j}^{g}\left|z_{j}(t)\right| e^{\omega t}-\frac{e^{\omega \sigma}}{\gamma} \sum_{j=1}^{n} c_{i j}^{+} L_{j}^{g} \\
& \quad \times\left|z_{j}\left(t-\sigma_{i j}(t)\right)\right| e^{\omega\left(t-\sigma_{i j}(t)\right)}\left(1-\sigma_{i j}^{\prime}(t)\right) \\
& +\frac{e^{\omega \tau}}{\rho} \sum_{j=1}^{n} \alpha_{i j}^{+} L_{j}^{h}\left|z_{j}(t)\right| e^{\omega t} \\
& \quad-\frac{e^{\omega \tau}}{\rho} \sum_{j=1}^{n} \alpha_{i j}^{+} L_{j}^{h}\left|z_{j}\left(t-\tau_{i j}(t)\right)\right| \\
& \quad \times e^{\omega\left(t-\tau_{i j}(t)\right)}\left(1-\tau_{i j}^{\prime}(t)\right)+\frac{e^{\omega \eta}}{\varrho} \sum_{j=1}^{n} \beta_{i j}^{+} \\
& \quad \times L_{j}^{k}\left|z_{j}(t)\right| e^{\omega t}-\frac{e^{\omega \eta}}{\varrho} \sum_{j=1}^{n} \beta_{i j}^{+} L_{j}^{h} \mid z_{j}(t
\end{aligned}
$$




$$
\begin{aligned}
& \left.-\eta_{i j}(t)\right) \mid e^{\omega\left(t-\eta_{i j}(t)\right)}\left(1-\eta_{i j}^{\prime}(t)\right) \\
& +\frac{e^{\omega \xi}}{\varsigma} \sum_{j=1}^{n} e_{i j}^{+} L_{j}^{l}\left|z_{j}(t)\right| e^{\omega t}-\frac{e^{\omega \xi}}{\varsigma} \sum_{j=1}^{n} e_{i j}^{+} L_{j}^{l} \\
& \left.\times\left|z_{j}\left(t-\xi_{i j}(t)\right)\right| e^{\omega\left(t-\xi_{i j}(t)\right)}\left(1-\xi_{i j}^{\prime}(t)\right)\right\} \\
& \leq \sum_{i=1}^{n}\left\{\omega W_{i}(t)+e^{\omega t}\left[-\left(a_{i}^{-}+\kappa_{i}^{-}\right)\left|z_{i}(t)\right|\right.\right. \\
& +\sum_{j=1}^{n} b_{i j}^{+} L_{j}^{f}\left|z_{j}(t)\right|+\sum_{j=1}^{n} c_{i j}^{+} L_{j}^{g}\left|z_{j}\left(t-\sigma_{i j}(t)\right)\right| \\
& +\sum_{j=1}^{n} \alpha_{i j}^{+} L_{j}^{h}\left|z_{j}\left(t-\tau_{i j}(t)\right)\right|+\sum_{j=1}^{n} \beta_{i j}^{+} L_{j}^{h} \mid z_{j}(t \\
& \left.\left.-\eta_{i j}(t)\right)\left|+\sum_{j=1}^{n} e_{i j}^{+} L_{j}^{l}\right| z_{j}\left(t-\xi_{i j}(t)\right) \mid\right] \\
& +\frac{e^{\omega \sigma}}{\gamma} \sum_{j=1}^{n} c_{i j}^{+} L_{j}^{g}\left|z_{j}(t)\right| e^{\omega t}-\frac{e^{\omega \sigma}}{\gamma} \sum_{j=1}^{n} c_{i j}^{+} L_{j}^{g} \\
& \times\left|z_{j}\left(t-\sigma_{i j}(t)\right)\right| e^{\omega\left(t-\sigma_{i j}(t)\right)}\left(1-\sigma_{i j}^{\prime}(t)\right) \\
& +\frac{e^{\omega \tau}}{\rho} \sum_{j=1}^{n} \alpha_{i j}^{+} L_{j}^{h}\left|z_{j}(t)\right| e^{\omega t}-\frac{e^{\omega \tau}}{\rho} \sum_{j=1}^{n} \alpha_{i j}^{+} L_{j}^{h} \\
& \times\left|z_{j}\left(t-\tau_{i j}(t)\right)\right| e^{\omega\left(t-\tau_{i j}(t)\right)}\left(1-\tau_{i j}^{\prime}(t)\right) \\
& +\frac{e^{\omega \eta}}{\varrho} \sum_{j=1}^{n} \beta_{i j}^{+} L_{j}^{h}\left|z_{j}(t)\right| e^{\omega t}-\frac{e^{\omega \eta}}{\varrho} \sum_{j=1}^{n} \beta_{i j}^{+} L_{j}^{h} \\
& \times\left|z_{j}\left(t-\eta_{i j}(t)\right)\right| e^{\omega\left(t-\eta_{i j}(t)\right)}\left(1-\eta_{i j}^{\prime}(t)\right) \\
& +\frac{e^{\omega \xi}}{\varsigma} \sum_{j=1}^{n} e_{i j}^{+} L_{j}^{l}\left|z_{j}(t)\right| e^{\omega t}-\frac{e^{\omega \xi}}{\varsigma} \sum_{j=1}^{n} e_{i j}^{+} L_{j}^{l} \\
& \left.\times\left|z_{j}\left(t-\xi_{i j}(t)\right)\right| e^{\omega\left(t-\xi_{i j}(t)\right)}\left(1-\xi_{i j}^{\prime}(t)\right)\right\} \\
& \leq \sum_{i=1}^{n}\left\{W _ { i } ( t ) \left[\omega-\left(a_{i}^{-}+\kappa_{i}^{-}\right)+\sum_{j=1}^{n} b_{i j}^{+} L_{j}^{f}\right.\right. \\
& +\frac{e^{\omega \sigma}}{\gamma} \sum_{j=1}^{n} c_{i j}^{+} L_{j}^{g}+\frac{e^{\omega \tau}}{\rho} \sum_{j=1}^{n} \alpha_{i j}^{+} L_{j}^{h} \\
& \left.\left.+\frac{e^{\omega \eta}}{\varrho} \sum_{j=1}^{n} \beta_{i j}^{+} L_{j}^{h}+\frac{e^{\omega \xi}}{\varsigma} \sum_{j=1}^{n} e_{i j}^{+} L_{j}^{l}\right]\right\} .
\end{aligned}
$$

From Assumption $A_{6}$, we can calculate the right limits of Lyapunov functional $V$ at impulsive moments $t_{k}$ as follows:

$$
\begin{aligned}
V\left(t_{k}^{+}\right)= & \sum_{i=1}^{n}\left\{W_{i}\left(t_{k}^{+}\right)+\frac{e^{\omega \sigma}}{\gamma} \sum_{j=1}^{n} c_{i j}^{+} L_{j}^{g}\right. \\
& \times \int_{t_{k}^{+}-\sigma_{i j}\left(t_{k}^{+}\right)}^{t_{k}^{+}} e^{\omega s}\left|z_{j}(s)\right| \mathrm{d} s+\frac{e^{\omega \tau}}{\rho} \sum_{j=1}^{n} \alpha_{i j}^{+}
\end{aligned}
$$

$$
\begin{aligned}
& \times L_{j}^{h} \int_{t_{k}^{+}-\tau_{i j}\left(t_{k}^{+}\right)}^{t_{k}^{+}} e^{\omega s}\left|z_{j}(s)\right| \mathrm{d} s \\
& +\frac{e^{\omega \eta}}{\varrho} \sum_{j=1}^{n} \beta_{i j}^{+} L_{j}^{h} \int_{t_{k}^{+}-\eta_{i j}\left(t_{k}^{+}\right)}^{t_{k}^{+}} e^{\omega s}\left|z_{j}(s)\right| \mathrm{d} s \\
& \left.+\frac{e^{\omega \xi}}{\varsigma} \sum_{j=1}^{n} e_{i j}^{+} L_{j}^{l} \int_{t_{k}^{+}-\xi_{i j}\left(t_{k}^{+}\right)}^{t_{k}^{+}}\left|z_{j}(s)\right| e^{\omega s} \mathrm{~d} s\right\} \\
& \leq \sum_{i=1}^{n}\left\{W_{i}\left(t_{k}^{-}\right)+\frac{e^{\omega \sigma}}{\gamma} \sum_{j=1}^{n} c_{i j}^{+} L_{j}^{g}\right. \\
& \times \int_{t_{k}^{-}-\sigma_{i j}\left(t_{k}^{-}\right)}^{t_{k}^{-}} e^{\omega s}\left|z_{j}(s)\right| \mathrm{d} s \\
& +\frac{e^{\omega \tau}}{\rho} \sum_{j=1}^{n} \alpha_{i j}^{+} L_{j}^{h} \int_{t_{k}^{-}-\tau_{i j}\left(t_{k}^{-}\right)}^{t_{k}^{-}} e^{\omega s}\left|z_{j}(s)\right| \mathrm{d} s \\
& +\frac{e^{\omega \eta}}{\varrho} \sum_{j=1}^{n} \beta_{i j}^{+} L_{j}^{h} \int_{t_{k}^{-}-\eta_{i j}\left(t_{k}^{-}\right)}^{t_{k}^{-}} e^{\omega s}\left|z_{j}(s)\right| \mathrm{d} s \\
& \left.+\frac{e^{\omega \xi}}{\varsigma} \sum_{j=1}^{n} e_{i j}^{+} L_{j}^{l} \int_{t_{k}^{-}-\xi_{i j}\left(t_{k}^{-}\right)}^{t_{k}^{-}}\left|z_{j}(s)\right| e^{\omega s} \mathrm{~d} s\right\} \\
& =V\left(t_{k}^{-}\right), \quad k \in \mathbb{Z}^{+} .
\end{aligned}
$$

In view of Assumption $A_{7}, 10$ and (11), we obtain

$$
D^{+} V(t) \leq 0
$$

which implies $V(t) \leq V(0)$ for all $t \geq 0$.

On the other hand, we have

$$
\begin{aligned}
V(0)= & \sum_{i=1}^{n}\left\{W_{i}(0)+\frac{e^{\omega \sigma}}{\gamma} \sum_{j=1}^{n} c_{i j}^{+} L_{j}^{g}\right. \\
& \times \int_{-\sigma_{i j}(0)}^{0} e^{\omega s}\left|z_{j}(s)\right| \mathrm{d} s+\frac{e^{\omega \tau}}{\rho} \sum_{j=1}^{n} \alpha_{i j}^{+} L_{j}^{h} \\
& \times \int_{-\tau_{i j}(0)}^{0} e^{\omega s}\left|z_{j}(s)\right| \mathrm{d} s+\frac{e^{\omega \eta}}{\varrho} \sum_{j=1}^{n} \beta_{i j}^{+} L_{j}^{h} \\
& \times \int_{-\eta_{i j}(0)}^{0} e^{\omega s}\left|z_{j}(s)\right| \mathrm{d} s \\
& \left.+\frac{e^{\omega \xi}}{\varsigma} \sum_{j=1}^{n} e_{i j}^{+} L_{j}^{l} \int_{-\xi_{i j}(0)}^{0}\left|z_{j}(s)\right| e^{\omega s} \mathrm{~d} s\right\} \\
\leq & \sum_{i=1}^{n}\left[1+\frac{e^{\omega \sigma}-1}{\omega \gamma} \sum_{j=1}^{n} c_{i j}^{+} L_{j}^{g}\right. \\
& +\frac{e^{\omega \tau}-1}{\omega \rho} \sum_{j=1}^{n} \alpha_{i j}^{+} L_{j}^{h} \\
& +\frac{e^{\omega \eta}-1}{\omega \varrho} \sum_{j=1}^{n} \beta_{i j}^{+} L_{j}^{h} \\
& \left.+\frac{e^{\omega \xi}-1}{\omega \varsigma} \sum_{j=1}^{n} e_{i j}^{+} L_{j}^{l}\right]\|\psi-\varphi\|_{0} .
\end{aligned}
$$


It is easy to check that

$$
\begin{aligned}
\|y(t)-x(t)\| & \leq V(t) e^{-\omega t} \leq V(0) e^{-\omega t} \\
& \leq M e^{-\omega t}\|\psi-\varphi\|_{0}, \quad t \geq 0
\end{aligned}
$$

where

$$
\begin{aligned}
M= & \sum_{i=1}^{n}\left[1+\frac{e^{\omega \sigma}-1}{\omega \gamma} \sum_{j=1}^{n} c_{i j}^{+} L_{j}^{g}\right. \\
& +\frac{e^{\omega \tau}-1}{\omega \rho} \sum_{j=1}^{n} \alpha_{i j}^{+} L_{j}^{h}+\frac{e^{\omega \eta}-1}{\omega \varrho} \sum_{j=1}^{n} \beta_{i j}^{+} L_{j}^{h} \\
& \left.+\frac{e^{\omega \xi}-1}{\omega \varsigma} \sum_{j=1}^{n} e_{i j}^{+} L_{j}^{l}\right]>0 .
\end{aligned}
$$

Therefore, the drive system (1) and the response system (5) are globally exponentially synchronized. The proof is complete.

Remark 2. Li et al. (2018b; 2018a) considered almost periodic synchronization and pseudo almost periodic synchronization for quaternion-valued cellular neural networks with time-varying delays, but the controllers in those works are only state feedback ones, not the state feedback and the impulsive controller. In addition, the networks considered in these works are deterministic, rather than fuzzy networks. Therefore, Theorem 2 is new.

\section{Numerical example}

In this section, an example is given to show the effectiveness of the result obtained in this paper.

Example 1. Consider the following fuzzy cellular neural networks as the drive system:

$$
\begin{aligned}
x_{i}^{\prime}(t)= & -a_{i}(t) x_{i}(t)+\sum_{j=1}^{2} b_{i j}(t) f_{j}\left(x_{j}(t)\right) \\
& +\sum_{j=1}^{2} c_{i j}(t) g_{j}\left(x_{j}\left(t-\sigma_{i j}(t)\right)\right) \\
& +\sum_{j=1}^{2} d_{i j}(t) \mu_{j}(t)+\bigwedge_{j=1}^{2} T_{i j}(t) \mu_{j}(t) \\
& +\bigwedge_{j=1}^{2} \alpha_{i j}(t) h_{j}\left(x_{j}\left(t-\tau_{i j}(t)\right)\right) \\
& +\bigvee_{j=1}^{2} \beta_{i j}(t) h_{j}\left(x_{j}\left(t-\eta_{i j}(t)\right)\right) \\
& +\bigvee_{j=1}^{2} S_{i j}(t) \mu_{j}(t)+I_{i}(t) .
\end{aligned}
$$

The corresponding response system is given by

$$
\begin{aligned}
y_{i}^{\prime}(t)= & -a_{i}(t) y_{i}(t)+\sum_{j=1}^{2} b_{i j}(t) f_{j}\left(y_{j}(t)\right) \\
& +\sum_{j=1}^{2} c_{i j}(t) g_{j}\left(y_{j}\left(t-\sigma_{i j}(t)\right)\right) \\
& +\sum_{j=1}^{2} d_{i j}(t) \mu_{j}(t)+\bigwedge_{j=1}^{2} T_{i j}(t) \mu_{j}(t) \\
& +\bigwedge_{j=1}^{2} \alpha_{i j}(t) h_{j}\left(y_{j}\left(t-\tau_{i j}(t)\right)\right) \\
& +\bigvee_{j=1}^{2} \beta_{i j}(t) h_{j}\left(y_{j}\left(t-\eta_{i j}(t)\right)\right) \\
& +\bigvee_{j=1}^{2} S_{i j}(t) \mu_{j}(t)+I_{i}(t)+u_{i}(t),
\end{aligned}
$$

and the controller is as follows:

$$
\begin{aligned}
u_{i}(t)= & \sum_{k=1}^{\infty} J_{i k}\left(z_{i}(t)\right) \delta\left(t-t_{k}\right)-\kappa_{i}(t) z_{i}(t) \\
& +\sum_{j=1}^{n} e_{i j}(t) l_{j}\left(z_{j}\left(t-\xi_{i j}(t)\right)\right),
\end{aligned}
$$

where $i=1,2$, and the coefficients are

$$
\begin{gathered}
f_{1}(x)=f_{2}(x)=0.3 \sin x, \\
g_{1}(x)=g_{2}(x)=0.2 \tanh x, \\
h_{1}(x)=h_{2}(x)=0.5(|x+1|+|x|-1), \\
l_{1}(x)=l_{2}(x)=0.4|x|,
\end{gathered}
$$

$$
\begin{aligned}
a_{1}(t) & =\sin \sqrt{2} t+3, & & a_{2}(t)=\cos 2 t+4, \\
I_{1}(t) & =0.1 \sin 2 t, & I_{1}(t) & =0.1 \sin \sqrt{3} t, \\
\mu_{1}(t) & =\sin t, & \mu_{2}(t) & =\cos t, \\
b_{11}(t) & =0.1 \sin 5 t, & b_{12}(t) & =0.2 \cos 2 t, \\
b_{21}(t) & =0.4 \sin t, & b_{22}(t) & =0.5 \cos t, \\
c_{11}(t) & =0.3 \sin 7 t, & c_{12}(t) & =0.1 \cos t, \\
c_{21}(t) & =0.4 \cos 3 t, & c_{22}(t) & =0.2 \sin \sqrt{2} t, \\
d_{11}(t) & =0.5 \sin \sqrt{2} t, & d_{12}(t) & =0.5 \sin 5 t, \\
d_{21}(t) & =0.4 \cos \sqrt{3} t, & d_{22}(t) & =0.5 \sin t, \\
\alpha_{11}(t) & =0.25 \cos t, & \alpha_{12}(t) & =0.5 \sin 2 t, \\
\alpha_{21}(t) & =0.6 \sin 5 t, & \alpha_{22}(t) & =0.3 \sin \sqrt{3} t, \\
\beta_{11}(t) & =0.2 \cos \sqrt{3} t, & \beta_{12}(t) & =0.1 \sin \sqrt{5} t, \\
\beta_{21}(t) & =0.3 \cos t, & \beta_{22}(t) & =0.45 \sin t, \\
T_{11}(t) & =0.3 \sin 2 t, & T_{12}(t) & =0.4 \cos t,
\end{aligned}
$$




$$
\begin{aligned}
T_{21}(t) & =\sin \sqrt{2} t, & & T_{22}(t)=\cos \sqrt{3} t, \\
S_{11}(t) & =0.5 \sin t, & & S_{12}(t)=0.6 \cos t, \\
S_{21}(t) & =\sin 3 t, & S_{22}(t) & =\sin \sqrt{3} t, \\
\kappa_{1}(t) & =\cos \sqrt{2} t+3, & \kappa_{2}(t) & =\sin t+4, \\
e_{11}(t) & =0.1 \sin \sqrt{2} t, & e_{12}(t) & =0.2 \sin t, \\
e_{21}(t) & =0.3 \sin 2 t, & e_{22}(t) & =0.2 \cos \sqrt{3} t, \\
\sigma_{i j}(t) & =0.4 \sin ^{2} t, & \tau_{i j}(t) & =0.2 \cos ^{2} t, \\
\eta_{i j}(t) & =0.3|\sin t|, & \xi_{i j}(t) & =0.5|\cos t| .
\end{aligned}
$$

By simple calculation, we obtain

$$
\begin{aligned}
& a_{1}^{-}=2, \quad \kappa_{1}^{-}=2, \quad a_{2}^{-}=3, \quad \kappa_{2}^{-}=3, \\
& L_{1}^{f}=L_{2}^{f}=0.3, \quad L_{1}^{g}=L_{2}^{g}=0.2, \\
& L_{1}^{h}=L_{2}^{h}=1, \quad L_{1}^{l}=L_{2}^{l}=0.4, \\
& b_{11}^{+}=0.1, \quad b_{12}^{+}=0.2, \quad b_{21}^{+}=0.4, \quad b_{22}^{+}=0.5, \\
& c_{11}^{+}=0.3, \quad c_{12}^{+}=0.1, \quad c_{21}^{+}=0.4, \quad c_{22}^{+}=0.2, \\
& d_{11}^{+}=0.5, \quad d_{12}^{+}=0.5, \quad d_{21}^{+}=0.4, \quad d_{22}^{+}=0.5, \\
& \alpha_{11}^{+}=0.25, \quad \alpha_{12}^{+}=0.5, \quad \alpha_{21}^{+}=0.6, \quad \alpha_{22}^{+}=0.3, \\
& \beta_{11}^{+}=0.2, \quad \beta_{12}^{+}=0.1, \quad \beta_{21}^{+}=0.3, \quad \beta_{22}^{+}=0.45, \\
& T_{11}^{+}=0.3, \quad T_{12}^{+}=0.4, \quad T_{21}^{+}=1, \quad T_{22}^{+}=1, \\
& S_{11}^{+}=0.5, \quad S_{12}^{+}=0.6, \quad S_{21}^{+}=1, \quad S_{22}^{+}=1, \\
& I_{1}^{+}=I_{2}^{+}=0.1, \quad \mu_{1}^{+}=\mu_{2}^{+}=1, \\
& e_{11}^{+}=0.1, \quad e_{12}^{+}=0.2, \quad e_{21}^{+}=0.3, \quad e_{22}^{+}=0.2, \\
& \sigma=0.4, \quad \tau=0.2, \quad \eta=0.3, \quad \xi=0.5, \\
& P_{1}=1.22, \quad P_{2}=2.04 \text {. }
\end{aligned}
$$

Then we obtain

$$
\max \left\{\frac{P_{1}}{a_{1}^{-}}, \frac{P_{2}}{a_{2}^{-}}\right\} \approx \max \{0.61,0.68\}=0.68<1,
$$

and therefore all the conditions of Theorems 11 are satisfied, so the drive system (12) has an almost periodic solution. Moreover, take $t_{k}=0.5+2(k-1) \pi, k \in \mathbb{Z}^{+}$ and impulsive functions $J_{i k}\left(z_{i}\right)=-\left(1+\frac{1}{3} \sin (2+k)\right) z_{i}$, $i=1,2, \omega=1, \gamma=0.6, \rho=0.8, \varrho=0.7, \varsigma=0.5$; then we get

$$
\begin{aligned}
\max _{i=1,2}\left\{\omega-\left(a_{i}^{-}+\kappa_{i}^{-}\right)+\sum_{j=1}^{n} b_{i j}^{+} L_{j}^{f}\right. \\
+\frac{e^{\omega \sigma}}{\gamma} \sum_{j=1}^{n} c_{i j}^{+} L_{j}^{g}+\frac{e^{\omega \tau}}{\rho} \sum_{j=1}^{n} \alpha_{i j}^{+} L_{j}^{h} \\
\left.+\frac{e^{\omega \eta}}{\varrho} \sum_{j=1}^{n} \beta_{i j}^{+} L_{j}^{h}+\frac{e^{\omega \xi}}{\varsigma} \sum_{j=1}^{n} e_{i j}^{+} L_{j}^{l}\right\}
\end{aligned}
$$

$$
\begin{aligned}
& \approx \max \{-0.5918,-0.8198\} \\
& =-0.5918<0 .
\end{aligned}
$$

Thus, Assumption $A_{7}$ is also satisfied. Therefore, the systems (12) and (13) can be globally exponentially synchronized (see Figs. 1-6).

\section{Conclusion}

In this paper, we considered the problem of almost periodic synchronization of fuzzy cellular neural networks with time-varying delays via state-feedback and impulsive control. By applying the Banach fixed point theorem, constructing a suitable Lyapunov function and designing a state-feedback and impulsive control, we obtained that the drive-response structure of fuzzy cellular neural networks with almost periodic coefficients realize exponential synchronization. We know that almost periodic synchronization for fuzzy cellular neural networks with time-varying delays via state-feedback and impulsive control is new. Our results are completely original and our methods can be used to study the problem of almost periodic and automorphic synchronization for other types of neural networks.

\section{Acknowledgment}

This work is supported by the National Natural Sciences Foundation of the People's Republic of China under the grants no. 11861072 and 11361072.

\section{References}

Abdurahman, A., Jiang, H. and Teng, Z. (2016). Finite-time synchronization for fuzzy cellular neural networks with time-varying delays, Fuzzy Sets and Systems 297: 96-111.

Aouiti, C., Gharbia, I.B., Cao, J., M'Hamdi, M.S. and Alsaedi, A. (2018). Existence and global exponential stability of pseudo almost periodic solution for neutral delay BAM neural networks with time-varying delay in leakage terms, Chaos Solitons \& Fractals 107: 111-127.

Aouiti, C., M'Hamdi, M.S., Cao, J. and Alsaedi, A. (2017). Piecewise pseudo almost periodic solution for impulsive generalised high-order Hopfield neural networks with leakage delays, Neural Processing Letters 45(2): 615-648.

Arik, S. (2002a). An analysis of global asymptotic stability of delayed cellular neural networks, IEEE Transactions on Neural Networks 13(5): 1239-1242.

Arik, S. (2002b). An improved global stability result for delayed cellular neural networks, IEEE Transactions on Circuits \& Systems I: Fundamental Theory \& Applications 49(8): 1211-1214.

Cai, Z., Huang, L., Guo, Z., Zhang, L. and Wan, X. (2015). Periodic synchronization control of discontinuous delayed networks by using extended Filippov-framework, Neural Networks 68: 96-110. 


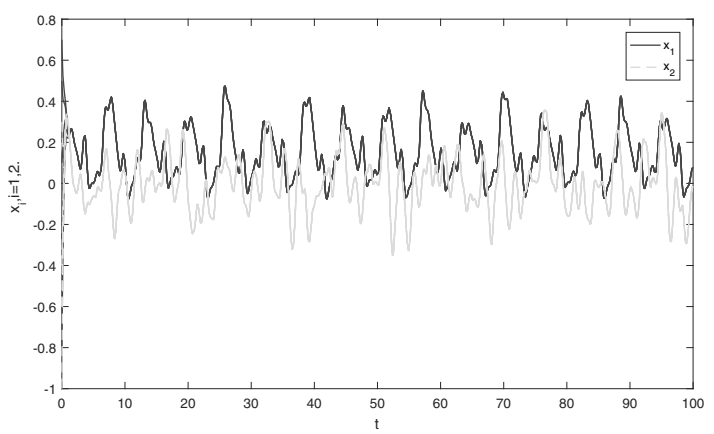

Fig. 1. States of $x_{1}$ and and $x_{2}$.

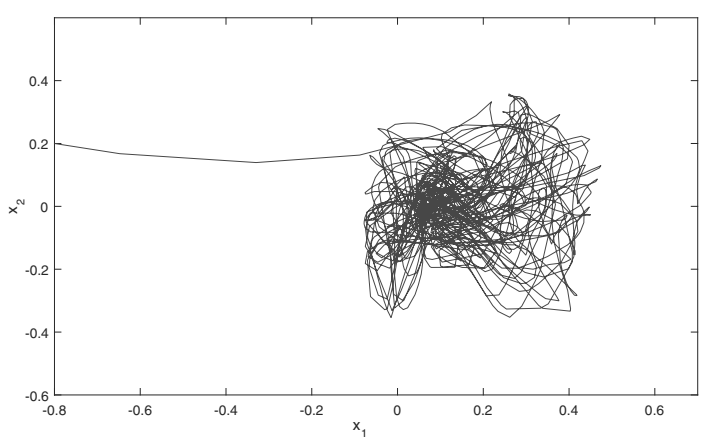

Fig. 2. Phase diagram of $x_{1}$ and and $x_{2}$

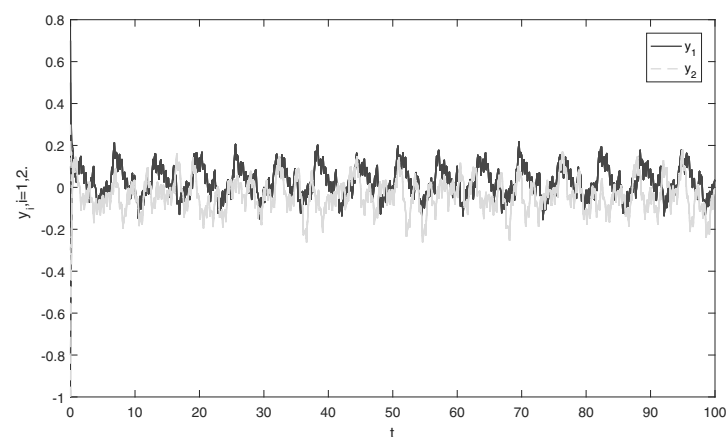

Fig. 3. States of $y_{1}$ and $y_{2}$.

Cao, J., Ho, D.W.C. and Yang, Y. (2009). Projective synchronization of a class of delayed chaotic systems via impulsive control, Physics Letters A 373(35): 3128-3133.

Cao, J., Li, H. and Ho, D.W.C. (2005). Synchronization criteria of Lur'e systems with time-delay feedback control, Chaos Solitons \& Fractals 23(4): 1285-1498.

Cao, J. and Liang, J. (2004). Boundedness and stability for Cohen-Grossberg neural network with time-varying delays, Journal of Mathematical Analysis and Applications 296(2): 665-685.

Diagana, T. (2013). Almost Automorphic Type and Almost Periodic Type Functions in Abstract Spaces, Springer, Cham.

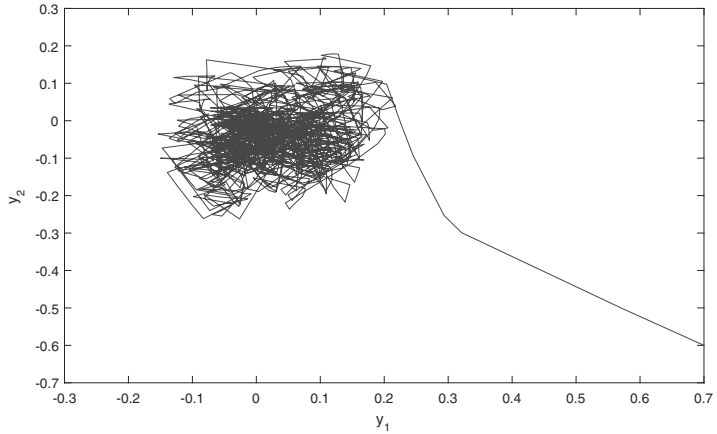

Fig. 4. Phase diagram of $y_{1}$ and $y_{2}$.

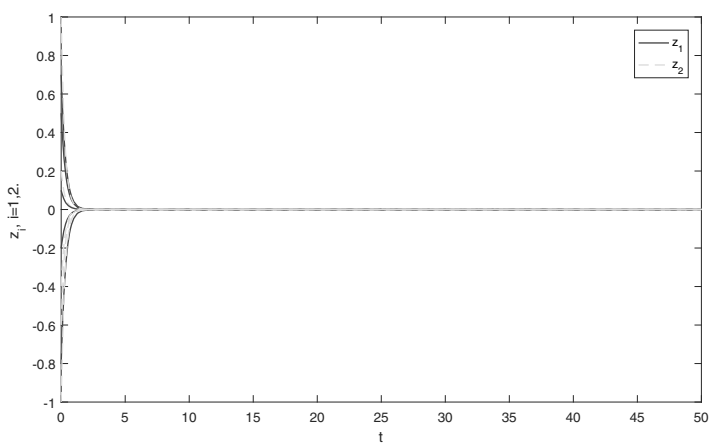

Fig. 5. Drive system (12) and its response system (13) are synchronized.

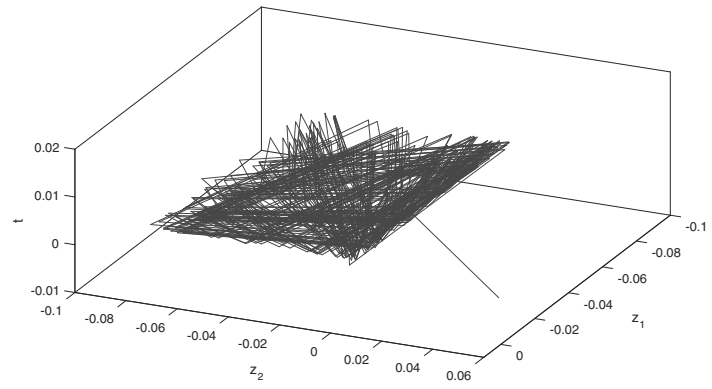

Fig. 6. Curves of $z$ in a 3-dimensional space for the synchronization case.

Ding, W. and Han, M. (2008). Synchronization of delayed fuzzy cellular neural networks based on adaptive control, Physics Letters A 372(26): 4674-4681.

Ding, W., Han, M. and Li, M. (2009). Exponential lag synchronization of delayed fuzzy cellular neural networks with impulses, Physics Letters A 373(8-9): 832-837.

Feng, X., Zhang, F. and Wang, W. (2011). Global exponential synchronization of delayed fuzzy cellular neural networks with impulsive effects, Chaos Solitons \& Fractals 44(1): 9-16.

Fink, A.M. (1974). Almost Periodic Differential Equations, Springer, Berlin. 
Guan, K. (2018). Global power-rate synchronization of chaotic neural networks with proportional delay via impulsive control, Neurocomputing 283: 256-265.

Heagy, J.F., Carroll, T.L. and Pecora, L.M. (1994). Experimental and numerical evidence for riddled basins in coupled chaotic systems, Physical Review Letters 73(26): 3528-3531.

Hong, H. (2014). Periodic synchronization and chimera in conformist and contrarian oscillators, Physical Review E 89(6): 1-37.

Hu, C., Yu, J., Jiang, H. and Teng, Z. (2010). Exponential stabilization and synchronization of neural networks with time-varying delays via periodically intermittent control, Nonlinearity 23(10): 2369-2391.

Huang, Z. (2017a). Almost periodic solutions for fuzzy cellular neural networks with multi-proportional delays, International Journal of Machine Learning and Cybernetics 28(4): 1-9.

Huang, Z. (2017b). Almost periodic solutions for fuzzy cellular neural networks with time-varying delays, Neural Computing and Applications 28(8): 2313-2320.

Li, Y., Chen, X. and Zhao, L. (2009). Stability and existence of periodic solutions to delayed Cohen-Grossberg BAM neural networks with impulses on time scales, Neurocomputing 72(7-9): 1621-1630.

Li, Y. and Fan, X. (2009). Existence and globally exponential stability of almost periodic solution for Cohen-Grossberg BAM neural networks with variable coefficients, Applied Mathematical Modelling 33(4): 2114-2120.

Li, Y., Li, B., Yao, S. and Xiong, L. (2018a). The global exponential pseudo almost periodic synchronization of quaternion-valued cellular neural networks with time-varying delays, Neurocomputing 303: 75-87.

Li, Y., Meng, X. and Ye, Y. (2018b). Almost periodic synchronization for quaternion-valued neural networks with time-varying delays, Complexity 2018, Article ID: 6504590.

Li, Y., Wang, H. and Meng, X. (2018c). Almost automorphic synchronization of quaternion-valued high-order Hopfield neural networks with time-varying and distributed delays, IMA Journal of Mathematical Control and Information: dny015, DOI:10.1093/imamci/dny015.

Li, Y. and Wang, C. (2013). Existence and global exponential stability of equilibrium for discrete-time fuzzy BAM neural networks with variable delays and impulses, Fuzzy Sets and Systems 217: 62-79.

Li, Y. and Zhang, T. (2009). Global exponential stability of fuzzy interval delayed neural networks with impulses on time scales, International Journal of Neural Systems 19(06): 449-456.

Lin, Y. and Zhang, Y. (2018). Synchronization of stochastic impulsive discrete-time delayed networks via pinning control, Neurocomputing 286: 31-40.

Long, S. and Xu, D. (2011). Stability analysis of stochastic fuzzy cellular neural networks with time-varying delays, Neurocomputing 69(14-15): 2385-2391.
Lu, J., Ho, D.W.C., Cao, J. and Kurths, J. (2013). Single impulsive controller for globally exponential synchronization of dynamical networks, Nonlinear Analysis: Real World Applications 14(1): 581-593.

Lu, X., Zhang, X. and Liu, Q. (2018). Finite-time synchronization of nonlinear complex dynamical networks on time scales via pinning impulsive control, Neurocomputing 275: 2104-110.

Pan, L. and Cao, J. (2011). Anti-periodic solution for delayed cellular neural networks with impulsive effects, Nonlinear Analysis: Real World Applications 12(6): 3014-3027.

Park, J.H. (2009). Synchronization of cellular neural networks of neutral type via dynamic feedback controller, Chaos Solitons \& Fractals 42(3): 1299-1304.

Pecora, L.M. and Carroll, T.L. (1990). Synchronization in chaotic systems, Physical Review Letters 64(8): 821-824.

Sen, M.D.L. (2006). Stability of impulsive time-varying systems and compactness of the operators mapping the input space into the state and output spaces, Journal of Mathematical Analysis and Applications 321(2): 621-650.

Stamov, G.T. (2012). Almost Periodic Solutions for Impulsive Differential Equations, Springer, Berlin.

Tang, Z., Park, J.H. and Feng, J. (2018a). Impulsive effects on quasi-synchronization of neural networks with parameter mismatches and time-varying delay, IEEE Transactions on Neural Networks and Learning Systems 29(4): 908-919.

Tang, Z., Park, J.H., Wang, Y. and Feng, J. (2018b). Distributed impulsive quasi-synchronization of Lur'e networks with proportional delay, IEEE Transactions on Cybernetics, 49(8): 3105-3115, DOI:10.1109/TCYB.2018.2839178.

Wang, W. (2018). Finite-time synchronization for a class of fuzzy cellular neural networks with time-varying coefficients and proportional delays, Fuzzy Sets and Systems 338: 40-49.

Wu, H., Li, R., Zhang, X. and Yao, R. (2015). Adaptive finite-time complete periodic synchronization of memristive neural networks with time delays, Neural Processing Letters 42(3): 563-583.

Xia, Y., Cao, J. and Cheng, S.S. (2007). Global exponential stability of delayed cellular neural networks with impulses, Neurocomputing 70(13-15): 2495-2501.

Xu, D. and Yang, Z. (2005). Impulsive delay differential inequality and stability of neural networks, Journal of Mathematical Analysis and Applications 305(1): 107-120.

Yang, H., Wang, X., Zhong, S. and Shu, L. (2018) Synchronization of nonlinear complex dynamical systems via delayed impulsive distributed control, Applied Mathematics and Computation 320: 75-85.

Yang, T. (2001). Impulsive Control Theory, Springer, Berlin.

Yang, T. and Yang, L.B. (1996). The global stability of fuzzy cellular neural network, IEEE Transactions on Circuits and Systems I: Fundamental Theory and Applications 43(10): 880-883. 
Yang, W., Yu, W., Cao, J., Alsaadi, F.E. and Hayat, T. (2017). Global exponential stability and lag synchronization for delayed memristive fuzzy Cohen-Grossberg BAM neural networks with impulses, Neural Networks 98: 122-153.

Yang, X., Cao, J. and Ho, D. W.C. (2015). Exponential synchronization of discontinuous neural networks with time-varying mixed delays via state feedback and impulsive control, Cognitive Neurodynamics 9(2): $113-128$.

Yuan, K., Cao, J. and Deng, J. (2006). Exponential stability and periodic solutions of fuzzy cellular neural networks with time-varying delays, Neurocomputing 69(13-15): 1619-1627.

Yuan, K., Fei, S. and Cao, J. (2014). Partial synchronization of the distributed parameter system with time delay via fuzzy control, IMA Journal of Mathematical Control and Information 31(1): 51-72.

Zhang, B., Deng, F., Xie, S. and Luo, S. (2018). Exponential synchronization of stochastic time-delayed memristor-based neural networks via distributed impulsive control, Neurocomputing 286: 41-50.

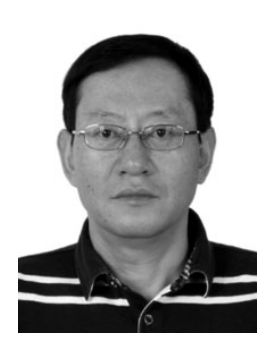

Yongkun $\mathbf{L i}$ received the $\mathrm{PhD}$ degree from Sichuan University, Chengdu, China. He is a professor of Yunnan University, Kunming, China. $\mathrm{He}$ is also the author or a co-author of more than 200 journal papers. His research interests include nonlinear dynamic systems, neural networks and applied mathematics.

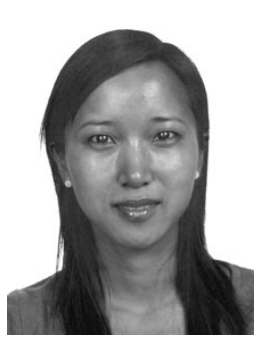

Huimei Wang received the MSc degree in applied mathematics from Yunnan University in 2007. Currently she is an assistant professor at Kunming University, China. She is working towards the $\mathrm{PhD}$ degree in applied mathematics at Yunnan University, China. Her research interests include differential equations and neural networks.

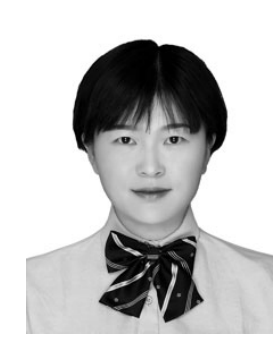

Xiaofang Meng received the BSc degree from Yunnan Agricultural University, Kunming, China, in 2012, and the MSc degree from Yunnan University, Kunming, in 2015. She is currently a $\mathrm{PhD}$ student at the Department of Mathematics, Yunnan University. Her present research interests include neural networks and nonlinear dynamic systems.

Received: 14 June 2018

Revised: 19 November 2018

Re-revised: 25 November 2018

Accepted: 22 February 2019 